\title{
COLLECTIVE SPATIAL COGNITION OF KIDS IN COMMUNITY MAPPINGS
}

\author{
A. Zare Zardiny ${ }^{1, *}$, F. Hakimpour ${ }^{1}$ \\ ${ }^{1}$ School of Surveying and Geospatial Engineering, University of Tehran, Tehran - (zare_zardiny, f.hakimpour)@ut.ac.ir
}

Commission VI, WG VI/4

KEY WORDS: Kids, Spatial Cognition, Community Mappings, Sketch maps, Qualitative GIS, Public Participatory GIS

\begin{abstract}
:
Due to the importance of spatial data in decision making and the cost of collecting these data, in recent years various communities have collaborated on spatial data collection. In these communities some expert and non-expert volunteers record their observations of a region in order to create a map. In these activities one of the most effective tools for recording observations is the sketch map. Due to ease of use and no need to comply with the common rules in Geospatial Information Systems, sketch maps can be drawn by a wide range of people. Because these maps are easy to use and following the GIS rules is not required, sketch maps can be drawn by a wide range of people. Although several studies have focused on raising the level of participation of ordinary people in the field of mapping, less attention has been given to the role of kids as an important part of the society. Hence, this paper including a field study examines the effect of collective spatial cognition of kids in a community mapping activity. For this purpose, the sketches drawn by some school kids are matched and then integrated together, and finally, the output of this process is compared with available metric maps. The results of this study show that despite the stringent conditions and the low age of the participants, the results have been beyond expectations. In this study, kids have provided several points of interest as well as more descriptive information of the region compared to the available data downloaded from OpenStreetMap and Google Maps. Therefore, the output of this study can be used to enrich the available metric map.
\end{abstract}

\section{INTRODUCTION}

During the human lifetime, place has always been one of the most effective parameters that effects on decision making, and as a result, collecting spatial data has been in great importance. The need for special equipment and experts has led to a limited range of people working in mapping fields. However, anyone can potentially collect and share data with regard to his/her daily interactions with the surrounding environment. This potential has led some non-expert people to start working in mapping fields. Community mapping is a term used to collect spatial data by a team of experts and also Non-expert volunteers. Community mapping can play an effective role, especially in cases which the collecting of spatial data is costly and time-consuming and does not require a high degree of data accuracy, or depending on the conditions, it is not possible to collect data with equipment (for example, in critic conditions).

Recording observations from a region by each individual, and then integrate these observations, are done in a community mapping activity. One of the most effective and most well-known tools of recording observations in community mapping is the sketch drawing. Sketch maps are a visualization of the spatial recognition of individuals from the real world, which originate from their observations and verbal connections. When people sketch a map, they are trying to draw some parts of the real world that they are most concerned with. The complexity of the surrounding world, the lack of complete knowledge and the different perceptions of individuals from surrounding environment and also, human errors at the time of drawing, cause the sketch maps to be accompanied by some distortions and heterogeneities. In the sketch maps, distances do not match the actual size. The angles are close to 90 degrees and the curve lines are drawn relatively straightforward. In addition, usually features with lower priority levels (from the point of view of individuals) are eliminated entirely or drawn in a simple form (Kitajima and Inoue, 2014). At first glance, based on the existence of these heterogeneities and distortions, the importance of sketch maps is not well defined, but in a more precise view, these maps can be used as a reliable method of providing and collecting spatial data (Blades, 1990). Drawing sketch map is a method that a very wide range of individuals, even people with no spatial expertise can collect and share spatial data. However, the use of potential in sketches drawn by the general public is considered in several studies, but the role of kids in the mapping activities has been less concerned. This concern shapes the basic idea of this paper. The goal of this paper is to raise the level of participation of individuals in the process of collecting the spatial data with a specific look at the role of kids. To reach this goal, this issue has been examined in a field study. In this study, 50 kids aged 8 to 10 years attending a school have been asked to draw sketches from the region around their school. In this activity, kids draw the sketches without the physical presence in the region of study and only based on their spatial cognition. In addition, kids are not trained about how to sketch and also, they do not have any kind of smart phone, online or paper maps from the region. The conditions considered in this activity are much more stringent than the circumstances commonly found in a community mapping activity. This article seeks to assess the quality of the

\footnotetext{
* Corresponding author
} 
output according to these conditions. In the following, the proposed method and the results are discussed.

This article has been organized in five main sections. In the first section, the related researches to the subject of this article are reviewed. Since understanding of the nature of the data in the sketch maps has significant effects on the matching process, hence in the second section, these types of data are investigated. The third section presents the proposed solution for matching and integrations the sketch maps. In the fourth section, the proposed solution are implemented and then evaluated. In the final section, while concluding the paper, the final results are expressed.

\section{PROPOSED METHOD}

Review of the researches on community mapping can be very helpful in understanding the role and importance of sketch maps in this type of activities. Poole in (2006) considers two main phases for the community mapping activities. The first phase is field data mapping in which individuals collect data by conducting interviews and drawing sketch map, and in the second phase, the process of digitizing these data is done. Poole in this article reports a community mapping activity aimed at providing land tenure mapping in Guyana Shield in Venezuela. Kanyara et al. in (2009), in a Community mapping activity, try to collects information on land rights and basic services in a region in Cambodia. They used sketch maps as an informal but useful method to minimize the cost of data collection and allowing more people to participate in this project. Wartmann and Purves in (2017) have been trying to answer this question whether using sketch maps can play an effective role in participatory mapping activity. The results of this exploratory case study, carried out with the participation of 29 people, show that in the interviews, information was collected from 156 POIs, and in the analysis of sketches, 74 POIs were extracted, of which only 23 were common. By examining these results, it is found that vegetation categories are highly diversified in language but seldom represented on maps, while more obviously anthropogenic features are represented on sketch maps. This means that sketch and interviewing can play a complementary role in participatory mapping activities. Despite the importance of sketch maps in community mappings and the role of the public in these activities, the role of children is less mentioned in the previous researches, and this is the subject matter that is the main idea behind this article.

\section{DATA IN SKETCH MAPS}

Data in sketch maps is more based on observations rather than measurements. Sketch maps generally include the relative and approximated positions of the features. These data lack georeference coordinates, and there is no complete matching between the distance and angles in the sketches and the metric space. Accordingly, these data can be investigated in a qualitative space. Among the existing data in sketch maps, routes can be considered as the most important type of data. Usually, in all sketch maps, it is possible to observe various displays of routes networks, and in some studies they are considered as one of the most important criteria for the data matching between the sketch maps and metric maps (Wang, 2009). In sketch maps, routes are not necessarily named, but they can be described in terms of proximity to certain POIs or their connections to the known routes. In this article, each route in the sketch maps is defined as a series of connected segments and the label of each segment is same as the related route. In addition, each route has a number of vertices. Among these vertices, only the first and the last node of each route and intersections are analysed in the matching process.
In addition, since people do not necessarily draw the roundabout as a closed loop, so in this process, roundabouts are also considered as junction. In the route network, by considering a predetermined direction as the $\mathrm{Y}$-axis of the local coordinate system in each sketch map, it is possible to define the first and the last nodes of routes and also the order of other nodes along the route. All of these segments and nodes form a labeled directional graph. Figure 1 depicts this graph and mentioned concepts.

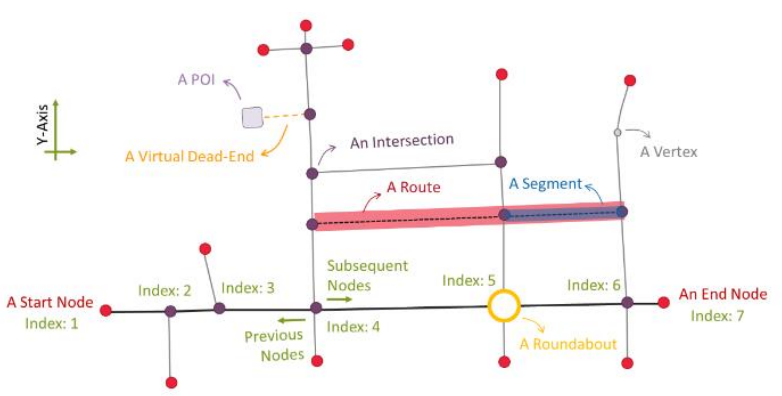

Figure 1. Data in a sketch maps in the form of a form a labeled directional graph

The second group of data existing in the sketch maps are regions and POIs that are drawn in adjacent to the route network. The importance of this type of data in relation to the sketch maps is that, on the one hand, they are more commonly known among the public, and on the other hand, usually all POIs depicted in a map have at least one name While all other data in sketch such as routes do not necessarily have names.

If the routes data are considered as a graph and the POI data are considered as a set of points in the vicinity of this graph, it is not possible to analyse all data simultaneously. Whiles people generally have all these data in their mind together. Therefore, the solution proposed here for this purpose is to integrate these data by adding the POIs data to the route network. For example, if a gas station is located in the vicinity of a route in sketch, this gas station can be replaced by a (virtual) dead-end. This process is just like that in reality instead of considering each of the POIs independently from the route Networks, their entrance path are considered as a part of the route network. In this way, all of the data in the sketch maps, such as route and POIs, can be entered into the data analysis process as a graph data model.

\section{PROPOSED SOLUTION}

This article concentrates on the construction of a synthetic map from sketches drawn by kids, and then comparing the integrated sketch with two available metric maps related to the region of study. To construct this map, the sketch with the highest number of features is considered as the basic sketch map. Subsequently, the process of matching and integrating the features extracted from each sketch map with the features in the basic sketch map is performed. Generally, to match the data in the sketch, it is necessary to measure the similarities between the features and then, the features with the most similarities are selected as the matched data. Therefore, the matching process can be considered as an optimization problem. In addition, as mentioned in the second section, different types of data in the sketch maps can be considered as a graph. Therefore, matching data in sketch maps can be defined in the form of the finding the Maximum Common Subgraph (MCS) of two graphs. Finding an MCS is a NPComplete problem (Garey and Johnson, 1978), (Thoresen, 2007) which is more appropriate to solved by non-deterministic methods. Considering the matching as an optimization problem 
and solving the MCS problem, the proposed method in this article is to use the Genetic Algorithm (GA). The matching process contains two phases. In the first phase of matching, the most similar features are selected as the matched features based on the similarity function. This matching process is done separately for each feature in the sketch maps, so this type of matching can be called the Partial Matching. Upon completion of the Partial Matching process, it may be possible to identify several corresponding features in basic sketch maps in exchange for each feature in other sketch maps. However, if all of the existing features in the sketch maps are considered together in the matching process, then a more acceptable result will be obtained for the search. Hence, after Partial Matching, it is necessary to choose the collection of features (in the basic sketch map) with the highest level of similarity to the sketch map. This process is called Global Matching. The important point here is the functions used to measure the similarity of the data in both of these two phases. These functions, which play the role of the target functions in the GA, include the parameters referred to below:

- Descriptive similarity: If two intersecting routes have labels in the sketch map, then the junction can be labeled based on their labels. Also, considering the roundabouts as junctions, their labels can be used to measure the descriptive similarity. Here, Levenshtein distance is used to measure the similarity of the labels for two routes. In accordance with equation 1, if $\mathrm{A}$ and $\mathrm{B}$ are the labels of two routes, in this case $\operatorname{LEV}_{A B}(i, j)$ calculates the distance between $i$-th character of A and j-th character of B (Levenshtein, 1966).

$$
\begin{aligned}
& \operatorname{LEV}_{A, B}(i, j) \\
& =\left\{\begin{array}{c}
\max (i, j) \\
\min \left\{\begin{array}{c}
L E V_{A, B}(i-1, j)+1 \\
L E V_{A, B}(i, j-1)+1 \\
L E V_{A, B}(i-1, j-1)+1\left(A_{i} \neq B_{j}\right)
\end{array}, \text { if } \min (i, j)=0\right.
\end{array}\right.
\end{aligned}
$$

According to this equation, if $|\mathrm{A}|$ and $|\mathrm{B}|$ are the number of characters $\mathrm{A}$ and $\mathrm{B}$, in this case the distance between two labels is obtained by calculating $L E V_{A, B}(|A|,|B|)$. Using this concept, the descriptive similarity of two junctions, $n_{i}$ and $n_{j}$ can be calculated in accordance with equation 2 :

$$
\begin{aligned}
& \text { DescriptiveSimilarity }\left(n_{i}, n_{j}\right) \\
& =1-\frac{\text { Levenshtein Distance }\left(l\left(n_{i}\right), l\left(n_{j}\right)\right)}{\operatorname{Max}\left(\left|l\left(n_{i}\right)\right|,\left|l\left(n_{j}\right)\right|\right)}
\end{aligned}
$$

In which, $l\left(n_{i}\right)$ and $l\left(n_{j}\right)$ are the labels of the junctions and $\left|l\left(n_{j}\right)\right|$ and $\left|l\left(n_{j}\right)\right|$ are the number of their characters.

- Ordering similarity: Considering a pre-defined direction, the Ordering Parameter (OP) can be defined as the ratio of the number of previous junctions to the total junctions located on the route. For example, in Figure 1 this ratio for the roundabout (located on the route with a dash-line style) is equal to $5 / 6$. Here, to calculate the ordering similarity of two junctions $n_{i}$ and $n_{j}$ the equation 3 are presented.

$$
\operatorname{OrderingSimilarity~}\left(n_{i}, n_{j}\right)=1-\frac{\left|O P\left(n_{i}\right)-O P\left(n_{j}\right)\right|}{100}
$$

- Shape similarity of the junctions: In order to describe the shape of a junction in this article, the valences (the number of adjacent connected nodes), the types and turns are used.
Here, the roundabouts are considered as a type of intersection with varying valences. Figure 2 shows the different shapes of the junctions depending on their valences. A relative turn (clockwise $(\mathrm{CW})$ or counterclockwise $(\mathrm{CCW})$ ) can be defined for Half-Cross junctions based on Figure 2.

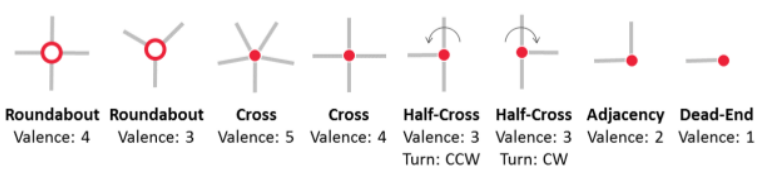

Figure 2. Different types of junctions with different valences, turns and types

Considering the valences, turns and types, if $n_{i}$ and $n_{j}$ are two junctions, then equation 4 can be used to measure the shape similarity of them:

$$
\begin{aligned}
& \text { ShapeSimilarity }\left(n_{i}, n_{j}\right) \\
& =c_{1} \text { ValenceSimilarity }\left(n_{i}, n_{j}\right)+c_{2} \text { TurnSimilarity }\left(n_{i}, n_{j}\right) \\
& +c_{3} \text { TypeSimilarity }\left(n_{i}, n_{j}\right)
\end{aligned}
$$

In which $c_{1}, c_{2}$ and $c_{3}$ are coefficients and

$$
\begin{aligned}
& \text { ValenceSimilarity }\left(n_{i}, n_{j}\right) \\
& =1-\frac{\left|\operatorname{Valence}\left(n_{i}\right)-\operatorname{Valence}\left(n_{j}\right)\right|}{\text { Max }_{\text {Valence }}(\text { Network })-\operatorname{Min}_{\text {Valence }}(\text { Network })} \\
& \text { TurnSimilarity }\left(n_{i}, n_{j}\right) \\
& = \begin{cases}1 \text { Valence }\left(n_{i}\right)=\operatorname{Valence}\left(n_{j}\right)=3 \& \operatorname{Turn}\left(n_{i}\right)=\operatorname{Turn}\left(n_{j}\right) \\
0 \quad \text { Otherwise }\end{cases} \\
& \text { TypeSimilarity }\left(n_{i}, n_{j}\right) \\
& =\left\{\begin{array}{cc}
1 \text { Type }\left(n_{i}\right)=\operatorname{Type}\left(n_{j}\right) \\
0 \quad \text { Otherwise }
\end{array}\right.
\end{aligned}
$$

- Proximity Similarity: In cases where several matched routes have been identified in the sketch map and the basic map, the proximity to these routes can be used for matching other routes' junctions. In order to calculate the proximity similarity, two characteristics of the relative distance and orientation of the junctions are considered. The distance is defined based on the number of junctions located on the shortest path between the two junctions. The relative parameter is defined according to the position of the junction in the left or right side of the matched route. Equation 8 describes the proximity similarity.

$$
\begin{aligned}
\text { ProximitySimilarity } & \left(n_{i}, n_{j}\right) \\
= & c_{4} \text { DistanceSimilarity }\left(n_{i,}, n_{j}\right) \\
+ & c_{5} \text { OrientationSimilarity }\left(n_{i}, n_{j}\right)
\end{aligned}
$$

In which $c_{4}$ and $c_{5}$ are coefficients and

$$
\begin{aligned}
& \text { DistanceSimilarity }\left(n_{i}, n_{j}\right) \\
& =1-\frac{\sum_{m=1}^{M} \mid \operatorname{NetworkDistance}\left(n_{i}, m\right)-\operatorname{NetworkDistance}\left(n_{j}, m\right)}{M * \operatorname{Max} \operatorname{Distance}(\operatorname{Network})} \\
& \text { OrientationSimilarity }\left(n_{i}, n_{j}\right) \\
& =\frac{\sum_{m=1}^{M}\left|\operatorname{Orientation}\left(n_{i}, m\right)==\operatorname{Orientation}\left(n_{j}, m\right)\right|}{M}
\end{aligned}
$$

Considering all the parameters, equation 11 can be used for measuring the node similarity: 


$$
\begin{aligned}
& \text { NodeSimilarity }\left(n_{i}, n_{j}\right) \\
& =\alpha_{1} \text { DescriptveSimilarity }\left(n_{i}, n_{j}\right) \\
& +\alpha_{2} \text { ShapeSimilarity }\left(n_{i}, n_{j}\right) \\
& +\alpha_{3} \text { OrderingSimilarity }\left(n_{i}, n_{j}\right) \\
& +\alpha_{4} \text { ProximitySimilarity }\left(n_{i}, n_{j}\right)
\end{aligned}
$$

In which $\alpha_{1}, \alpha_{2}, \alpha_{3}$ and $\alpha_{4}$ are coefficients.

Now, with regard to the similarity of nodes, the equation 12 can be used to measure the similarity of two routes $r_{1}$ and $r_{2}$ :

$$
\operatorname{Similarity}\left(r_{1}, r_{2}\right)=\sum_{s=0}^{N_{1}} M A X_{t=o}^{N_{2}}\left(\operatorname{NodeSimilarity}\left(n_{s}, n_{t}\right)\right)
$$

By defining the similarity function, the process of calculating the similarity between a feature in one sketch map and several random features (candidates) in the basic sketch map (as an initial population) begins. Then by using the elitism strategy, the candidates with the highest level of similarity are chosen as the parents for producing the new generation. To produce the next generation is done using the Mutation and Crossover operators. This process continues until the search result remains unchanged. Upon completion of the Partial Matching, several features in the basic sketch map may be selected for each feature in the sketch map. Hence, this result is not acceptable for matching. To improve the accuracy of the final result, in the next step, the Global Matching is performed on the results. What Global Matching is looking for to find a set of matched features identified in the basic sketch map with the highest level of correspondence to the entire of features in the sketch map The similarity function used here is defined as the ratio of the number of matched nodes between the two graphs to the total number of nodes in the basic sketch map. Other steps are similar to the Partial Matching process.

After completing the data matching of each sketch map with the basic sketch map, the integrating process should be performed on the data in sketch maps and the data in the basic sketch map. As shown in Figure 3, when between two nodes that create an edge in one graph, there is at least one node in the second graph, then the first edge does not appear on the output of the integration process and the split form is replaced. Here, the order of nodes along the routes is used to measure the length of an edge and in the integration process, the shorter routes is removed from the output. For example, in integration process of two route networks, shown in Figure 3, the route $\mathrm{AB}$ is replaced by $\mathrm{AC}$ and CB.

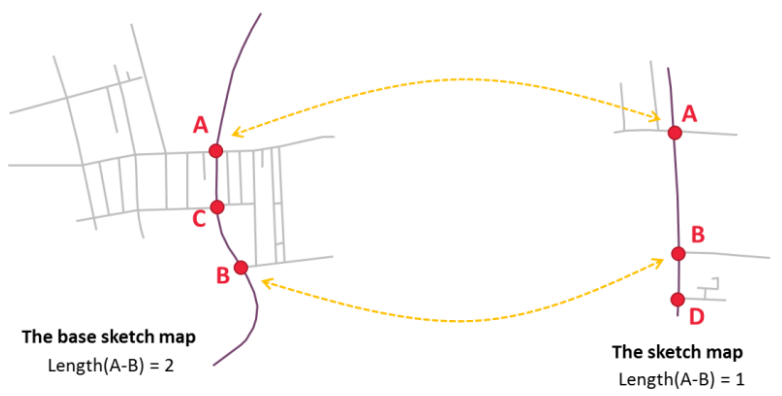

Figure3. Integration of routes in sketch maps

The segments that exist exactly in sketch map and the basic map, do not make geometric change in the integration process. However, these segments can increase the chance of their corresponding segments. Segments that exist in the sketch map but their splitted shaped exist in the basic map, have no effect on the integration process, because they do not add more information to the basic map. Segments that are in sketch map but not in the base map are also added to the basic map with minimal chance. The segments that are in the basic map and contains segments from sketch map are splitted and the least possible chance for the new segments is considered. The chance of each segment indicates the degree of certainty of that segment for appearing in the final result of the integration process.

\section{IMPLEMENTATION AND EVALUATION}

In this paper, in order to simulate a Community Mapping activity, 50 students aged 8 to 10 who study at one of the primary schools in Yazd (Zomorodi School) are asked to sketch a map from the area around their school in about 30 minutes. In this process, 50 sketches were obtained. The total number of routes drawn in these sketches is 256 , and of these routes, 62 routes are named. There are also 157 junctions and 45 roundabouts. The sketch maps also contain information of 195 POIs.

The implementation process begins by selecting the sketch map with the most number of features (as the basic sketch map), and then the process of matching and integration the data is performed for each sketch. The final output is shown in the Figure 4.

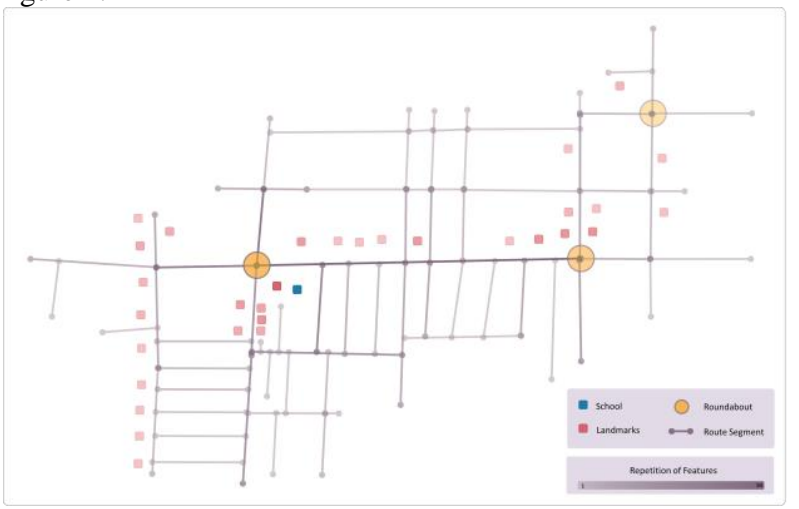

Figure 4. Sample output of the implementation

In order to evaluate the quality of the output, the generated map is compared with metric maps downloaded from OSM and Google Maps. This comparison is done manually and based on parameters such as the number of routes, number of POIs and number of the matched features. Figure 5 shows the results of this comparison. 


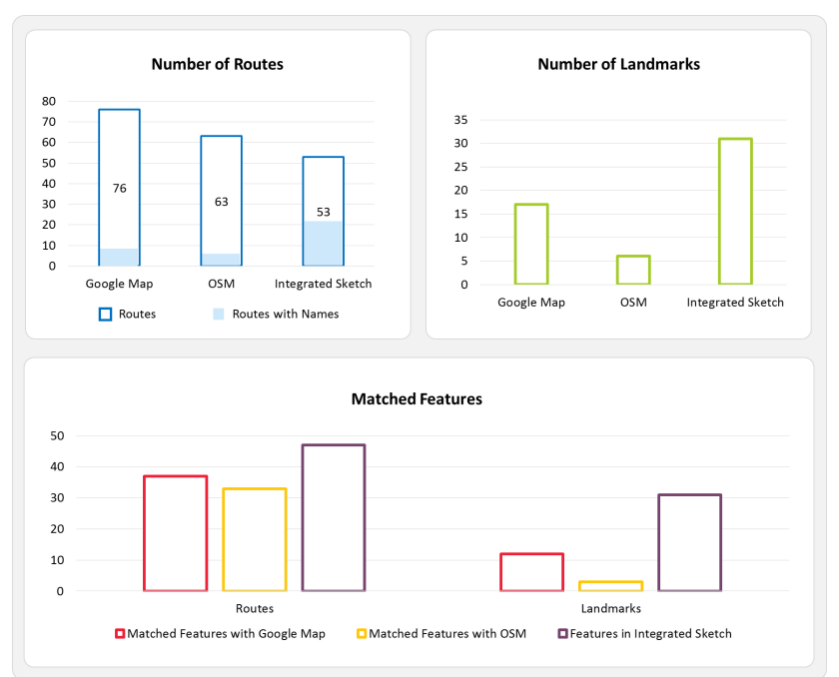

Figure 5. Comparison of the integrated sketch maps with OSM and Google Maps

According to Figure 5, although the number of routes drawn on the output is lower than those available on OSM and Google Maps, 39\% of these routes (in the sketch) are named. This amount is about $8 \%$ and $10 \%$ for OSM and Google Maps, respectively. There are also more POIs in the sketch maps. When the number of features in sketch maps is compared with the number of matched features between the maps, it can be concluded that some parts of these data are features that are not present in OSM and Google Maps. Therefore, descriptive and geometric enrichment of these metric maps with regard to the output of this study can be considered.

\section{CONCLUSION}

In this paper, by simulating a community mapping activity with kids, we tried to examine the role of kids in spatial data collecting activities. In this activity, conditions such as age limitations, lack of trainings for kids, lack of physical presence on site and the inability of using special equipment is considered. While in a real community mapping activity, the training is very important. Also, a group of expert and non-expert people work together on site. In addition, if possible, equipment such as smart phones, handheld GPS receivers, handhelds or laser meters, as well as online or paper maps are available to individuals. At first glance, it may seem that all the stringent conditions considered in this study can seriously reduce the output quality, but the results of this study have been beyond expectations. What can be derived from the integration of the sketch drawn by kids can be used in descriptive and geometric enrichment of available metric maps. Kids can play a complementary role in public GIS processes, such as the spatial data collecting, due to their different knowledge and perceptions of the world around them, as well as different mental engagements and concerns. Children can share their stories and experiences about the places where they live by picturing their emotions such as feeling secure, happiness or fear on the map. Their specific and different imagination of the world around them can be considered in different fields related to urban planning and this topic is one of the suggested topics for future researches.

\section{ACKNOWLEDGEMENT}

The sketches used in this paper are drawn by students in Zomorodi School in Yazd. We thank Ms.Mirkhalili and her colleagues for the help of providing these sketches.

\section{REFERENCES}

Kitajima, R., Inoue, U., 2014. Transformation of a Sketch Map Laying over a Metric Map on the Web. Lecture Notes on Software Engineering, Vol. 2, No. 3

Blades, M., 1990. The Reliability of Data Collected from Sketch Maps. Journal of Environmental Psychology, 10 (4), 327-339

Poole, P., 2006. Is there Life after Tenure Mapping? Participatory learning and action, No. 54, Pages: 41-49

Kanyara, S., Dara, O., Sherchan, D., 2009. Community Mapping Training Manual. Centre on Housing Rights and Evictions (COHRE), Asia \& Pacific Programme, CAMBODIA

Wartmann, F. M., Purves, R. S., 2017. What's (Not) on the Map: Landscape Features from Participatory Sketch Mapping Differ from Local Categories Used in Language, Land 2017, 6, 79; doi:10.3390/land6040079

Wang, J., 2009. How Human Schematization and Systematic Errors Take Effect on Sketch Map Formalizations. Master Thesis, Institute for Geoinformatics, University of Munster

Garey, M. R., Johnson, D. S., 1978, Computers and Intractability: A Guide to the Theory of NP-Completeness, W. H. Freeman \& Co. New York, NY, USA, ISBN: 0716710455

Thoresen, S., 2007. An Efficient Solution To Inexact Graph Matching With Application To Computer Vision, Department of Computer and Information Science, Norwegian University of Science and Technology, ISBN 978-82-471-3604-1

Levenshtein, V. I., 1966. Binary codes capable of correcting deletions, insertions, and reversals. Soviet Physics Doklady. 10 (8). pp. 707-710. 